\title{
Soberanía alimentaria y otras soberanías: el valor de los bienes comunes
}

\author{
Food Sovereignty and Other Sovereignties: \\ The Value of the Commons
}

\author{
Giovanna Micarelli* \\ Pontificia Universidad Javeriana
}

\begin{abstract}
RESUMEN
Si la realización del derecho a la alimentación depende en gran medida de la soberanía alimentaria, como ha sido recientemente reconocido, este artículo plantea que existe una relación íntima entre soberanía alimentaria, soberanía territorial y derecho a los bienes comunes. Mediante un estudio etnográfico del papel de la comida en construcciones socionaturales no dualistas, el artículo cuestiona la conceptualización economicista de los bienes comunes como recursos y propone ver "comunidades" y "recursos" como parte de un proceso de construcción simultánea, y por lo tanto inseparables. En diálogo con teorizaciones indígenas del concepto de soberanía, el texto busca expandir el concepto de soberanía alimentaria con el cual contribuir a una noción plural del derecho a la alimentación.
\end{abstract}

Palabras clave: soberanía alimentaria, bienes comunes, ontologías no dualistas.

\begin{abstract}
$\overline{\text { ABSTRACT }}$
If the realization of the right to food depends to a large degree on food sovereignty, as it has been recently recognized, this article argues that there is an intimate relationship between food sovereignty, territorial sovereignty, and the right to the commons. Through an ethnographic approach to the role of food in non-dualistic socio-natural constructions the article questions the economicist conceptualization of common goods as resources, and it proposes to see "communities" and "resources" as part of a process of simultaneous construction, and therefore inseparable. Dialoguing with indigenous theorizations of the concept of sovereignty, the article seeks to expand the notion offood sovereignty by which to contribute to a pluralist notion of the right to food.
\end{abstract}

Keywords: food sovereignty, commons, nondualistic ontologies.

\footnotetext{
Doctora en Antropología de la Universidad de Illinois. Profesora del Departamento de Antropología de la Pontificia Universidad Javeriana e investigadora del Centro de Estudios Sociales (Universidad de Coimbra). Su publicación más reciente es el libro Indigenous Networks at the Margins of Development (Bogotá: Universidad Javeriana, 2015). giomica@gmail.com.
} 
¡En el comer está la vida! Anciano uitoto, Amazonía colombiana

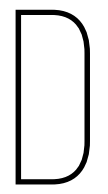

iversos movimientos alimentarios globales están llamando la atención sobre las limitaciones del modelo de seguridad alimentaria para garantizar el derecho a la alimentación de todos. En su lugar, proponen el modelo de la soberanía alimentaria para rediseñar, radical y democráticamente, los sistemas alimentarios. En un sentido amplio, el paradigma de la soberanía alimentaria reconoce que para garantizar el derecho a la alimentación no basta con centrarse en la disponibilidad y el acceso a los alimentos, sin tocar la cuestión de quién y cómo se producen. Mientras que, en su definición original de 1996, el movimiento internacional La Vía Campesina se refiere a la soberanía alimentaria como el "derecho de los pueblos y países a definir sus políticas agrícolas y alimentarias sin dumping ${ }^{1}$ frente a países terceros” (LVC 1996), más recientemente, la soberanía alimentaria ha adquirido el significado más radical del

[...] derecho de los pueblos a controlar sus propias semillas, tierras, agua y producción de alimentos [...] a través de una producción local, autónoma (participativa, comunitaria y compartida) y culturalmente apropiada, en armonía y complementación con la Madre Tierra. (CIPALC. Comité Internacional para la Soberanía Alimentaria-Coordinación Regional América Latina y el Caribe 2012)

Ante la mercantilización transnacional de los sistemas alimentarios, en el 2013 La Vía Campesina reconoció además que la protección de los bienes comunes se encuentra en el corazón de la soberanía alimentaria (LVC 2013). Esta transformación puede ser estratégica para ampliar las bases del consenso, pero al mismo tiempo revela diferentes órdenes de valor y formas alternativas de concebir los vínculos entre alimentos y comunidades.

Las conceptualizaciones más recientes de la soberanía alimentaria ponen énfasis en las redes de subsistencia y los sistemas de conocimiento locales, un punto que fue bien resumido por la investigadora y activista mexicana Silvia Ribeiro (2007), en el Foro para la Soberanía Alimentaria de Nyéléni, cuando señaló que "aquellos que realmente tienen los conocimientos necesarios para la soberanía alimentaria son los campesinos, los pescadores de pequeña escala, los pastores, las personas que pueden trabajar y vivir en el bosque”. El cambio de

1 La práctica de exportar productos a un precio inferior al costo de producción gracias a subvenciones. 
enfoque desde los agricultores como productores de alimentos a los agricultores como poseedores de conocimiento y gestores de sistemas agroecológicos sugiere que no solo hay que defender los recursos biodiversos sino sistemas culturales enteros. El énfasis también se amplía desde la tierra al territorio, intensificando las luchas mediante una politización del sentido (Porto-Gonçalves 2009). De hecho, las declaraciones de La Vía Campesina van precisando la soberanía alimentaria como el derecho de los pequeños agricultores a producir alimentos "en sus propios territorios” (LVC 1996). Esta organización insiste en que la soberanía alimentaria supone el "cuidado y uso sostenible de la tierra, el agua y las semillas con el fin de preservar la biodiversidad”; que está basada en "el derecho de los agricultores a utilizar libremente y proteger los recursos genéticos que han desarrollado" y en "defender sus territorios de las acciones de las empresas transnacionales” (Nyéléni 2007). Sin embargo, a pesar del claro énfasis en que los pueblos tengan control de sus bienes comunes en sus territorios, las relaciones complejas entre soberanía alimentaria, bienes comunes y el ejercicio comunitario de la soberanía territorial quedan todavía en gran medida inadvertidos. Prestar atención al significado de estas relaciones es crucial, ya que es esencialmente a través de estos significados que las comunidades y movimientos sociales articulan su resistencia y reclaman su autonomía.

El presente texto opta por la diferencia como punto de partida de esta tarea. Cultiva la idea de que una mirada atenta a lo divergente puede dejar emerger sensibilidades alternas desde las cuales poner en cuestión y desaprender las concepciones hegemónicas de soberanía, territorio y propiedad, y de esta manera ampliar las posibilidades del concepto de soberanía alimentaria para producir una transformación en el campo de los derechos. La presente reflexión teórica, necesariamente tentativa, se sustenta en mi trabajo de investigación con algunos pueblos indígenas de la Amazonía ${ }^{2}$ y se retroalimenta con un proyecto de investigación colectiva en curso en cuatro regiones de Colombia (La Guajira, Chocó, Nariño y Amazonas) ${ }^{3}$. También dialoga con, y busca articular, varias aproximaciones teóricas, entre las cuales está el planteamiento de Boaventura de Sousa

2 Desde 1995 he adelantado procesos de investigación tanto académica como comunitaria con los pueblos indígenas shipibo (Ucayali, Perú), muinane, bora, muina-murui, andoke, nonuya, miraña (del conjunto interétnico Gente de Centro), yucuna y tikuna (Colombia).

3 Proyecto "Food Sovereignty and Community's Valuation of Common Good" (mayo del 2017-febrero del 2018). Investigadores: Giovanna Micarelli (investigadora principal), Claudia Cáceres, Paula Bak, Luis Alberto Suárez. Financiación de la Pontificia Universidad Javeriana, Bogotá, convocatoria "Laudato Si': sobre el cuidado de la casa común". 
Santos sobre la necesidad de aprender de las epistemologías del sur ${ }^{4}$, las conceptualizaciones más recientes sobre los comunes y la vertiente teórica denominada giro ontológico, cuya genealogía ha sido influenciada de manera significativa por la antropología de las sociedades amerindias. Esta última, en particular, ha prestado paulatina atención a aquellas construcciones socionaturales indígenas que desafían la dicotomía entre sociedad y naturaleza instituida por la modernidad occidental, arrojando luz también sobre distintas nociones y prácticas de la propiedad implicadas por estas percepciones y modos de existencia divergentes. Este artículo toma posición desde estas perspectivas alternas sobre el mundo y las relaciones que lo constituyen y busca expandir el concepto de soberanía alimentaria mostrando la relación íntima que existe entre la soberanía alimentaria, el derecho a los bienes comunes y las formas locales de concebir y ejercer la soberanía territorial. Si bien esta relación es tejida por significados y prácticas culturales, este cambio de perspectiva no se limita a documentar la diversidad de percepciones culturales en torno al alimento, sino que tiene un objetivo más amplio, epistemológico y político al mismo tiempo. Pensar la soberanía alimentaria desde otras construcciones socionaturales puede proporcionar una serie de aprendizajes de relevancia global. En primer lugar, permite deshacer la dicotomía sujeto/objeto que ha estructurado tanto la conceptualización de los bienes comunes como la definición de los derechos fundamentales. En segundo lugar, sugiere rutas para tratar de teorizar la soberanía más allá de la noción clásica del Estado-nación, en términos de diferentes construcciones del mundo y de las responsabilidades que estas conllevan. El último objetivo de esta reflexión es asentar el derecho fundamental a la dignidad en el reconocimiento de las diferentes percepciones ontológicas y culturales en las cuales los derechos toman sentido, para finalmente ampliar el horizonte de las posibilidades disponibles o imaginables de resistencia frente al despojo de bienes alimentarios comunes como el agua, las semillas y los paisajes.

Entre el 2013 y el 2015 realicé una estancia posdoctoral en el Centro de Estudios Sociales dirigido por Boaventura de Sousa Santos en Coimbra y tuve la oportunidad de participar en su proyecto "Alice: espejos extraños, lecciones imprevistas". 


\section{El "valor" de los comunes: recursos versus colectividades}

Siempre más ubicados en el corazón de las luchas socioambientales globales, los bienes comunes ${ }^{5}$ establecen un nuevo discurso político sobre las condiciones necesarias para promover la justicia social, la sostenibilidad y una vida digna para todos, temas planteados por una variedad de movimientos sociales que se oponen al crecimiento capitalista (De Angelis 2010). Estas luchas involucran una disputa del sentido; como afirma la jurista Maria Rosaria Marella (2012), "[l]a contranarrativa de los bienes comunes permite desarticular los derechos de propiedad y reconectar lo que es legal a lo que es (ilegal pero percibido como) legítimo y justo" (3). De Angelis (2010) resume los elementos que integran la discusión actual sobre los bienes comunes:

Los comunes [commons] no son simplemente recursos que compartimos; la conceptualización de los bienes comunes implica tres cosas al mismo tiempo. En primer lugar, todos los comunes implican algún tipo de fondo común de recursos, entendido como medio no mercantilizado de cumplir las necesidades de la gente. En segundo lugar, los bienes comunes son necesariamente creados y mantenidos por las comunidades; este por supuesto es un término y un tema muy problemático, pero tenemos que pensar en ello. Las comunidades son grupos de comuneros que comparten estos recursos y que definen por sí mismos las reglas para acceder a ellos y poder utilizarlos. [...] Además de estos dos elementos - el conjunto de recursos y el conjunto de comunidades- el tercer y más importante elemento en términos de la conceptualización de los comunes es el proceso social que crea y reproduce los comunes: commoning en inglés. (S. p.)

Una perspectiva provocadora al respecto es ofrecida por el jurista Stefano Rodotà (2012), quien afirma que los bienes comunes son aquellos que son necesarios para hacer efectivos los derechos fundamentales de las personas, y por lo tanto no pueden ser considerados como un objeto de mercado. Al proponer una noción de bienes comunes como derecho fundamental, esta definición también cuestiona la idea de bienes comunes como recursos compartidos que son gestionados

5 La manera más simple de contrastar los bienes públicos y los comunes es preguntarse si un recurso en particular requiere la gestión como un mandato social o es una expresión de mutualidad social y colaboración. Es decir, si su propiedad es mantenida por el Gobierno o por la gente. Público pasó de significar la autoridad de una comunidad a administrar sus recursos locales, a la autoridad del Gobierno central a quien hemos cedido el control de estos recursos, que luego pueden ser privatizados (véase Quilligan 2012). 
por una comunidad de referencia - noción suscrita principalmente por los economistas - y desarticula la dicotomía sujeto/objeto que la fundamenta. Al ir más allá del reconocimiento de la Naturaleza como sujeto de derechos (Gudynas 2010), esta definición del derecho a los bienes comunes desdibuja el lindero entre una comunidad y los recursos de los cuales esta depende para el sustento y la supervivencia. A su vez, constatar que existe una relación inherente entre los bienes comunes y la realización de los derechos fundamentales requiere preguntar cómo traducen diferentes culturas esta idea en realidad.

Una posible traducción intercultural del concepto de commoning nos lleva a aquello de comunalidad acuñado por los intelectuales indígenas Floriberto Díaz y Jaime Martínez Luna, de las etnias mixe y zapoteca de México, respectivamente. La comunalidad abarca una serie de valores y prácticas que son expresión y reconocimiento de la pertenencia a lo colectivo: la visión del territorio como organismo viviente, la interdependencia, el trabajo colectivo, la autoridad como servicio, el consenso en asamblea y otras formas de hacer la vida en común. Estos valores y prácticas intervienen de manera sustancial en la evaluación crítica del comportamiento y en la construcción de acuerdos entre individuos y colectividades. De allí que sea necesario ver la relación entre "comunidades” y “comunes” más allá de las instituciones y las estrategias para la resolución de dilemas sociales - temas en que se ha concentrado el trabajo fundamental de Ostrom (1990) - y comprender que la eficacia y sostenibilidad de esta relación reside ante todo en la creación de marcos culturales ontoepistemológicos de referencia. De hecho, las normas no surgen en un vacío, sino que reflejan construcciones particulares del mundo en el que valores, sentimientos y expectativas son negociados en la práctica por los miembros de una colectividad.

El choque entre diferentes percepciones de los recursos naturales ha sido descrito por la ecología política como un choque entre diferentes "lenguajes de valoración”, cuya movilización en los procesos de resistencia contra el acaparamiento de los recursos confronta la hegemonía semántica que define el valor en términos exclusivamente monetarios (Martínez-Alier 2002; O’Connor y Spash 1999). Como ha sido evidenciado: "La evaluación de objetos bajo diferentes descripciones invoca no solo diferentes prácticas y perspectivas, sino también diferentes criterios y estándares para la evaluación asociados a estos. Lo que presupone es un pluralismo de valores” (Martínez-Alier, Munda y O’Neill 1998, 280). Esta observación puede ser llevada más lejos si, en vez de una perspectiva 
ontológica que ve los recursos como objetos hacia los cuales se dirige la evaluación, adoptamos como marco de referencia una perspectiva en que los "recursos" son parte integrante de un tejido que incluye al ser humano y del cual ambos dependen. El valor, lejos de ser un atributo asociado a "cosas" distintas del sujeto que evalúa, emerge de esta relación y, a su vez, reafirma los términos sobre los cuales se sostiene una construcción socionatural particular. La antropología ha recalcado que el valor es una construcción social y no puede ser reducido a términos económicos (Graeber 2001; Parry y Bloch 1989). Sin embargo, reconocer la existencia de diferentes regímenes de valor (Appadurai 1986) no es suficiente: es preciso deshacer la separación entre valor y valores, y entender los procesos de valoración desde los mundos simbólicos y prácticos construidos por los miembros de un grupo social: sus cosmologías. Las formas en que los recursos son valorados por las comunidades que basan en ellos su sustento envuelven -aunque tácitamente-concepciones de lo humano, la naturaleza, la vida, el conocimiento y la intersubjetividad, invocando, por lo tanto, criterios morales, sociales, ontológicos, epistemológicos y ecológicos, además de económicos. Preocupadas por cómo dar sentido al mundo, estas ideas no son inmunes a las turbulencias de la vida y de la historia. Como nos recuerda Barth (1987, 84), las cosmologías están siempre en fabricación y para entenderlas hay que dar cuenta de su producción en el contexto político con el que dialogan. Es más: al constituir distintos órdenes de lo valioso (Stark 2011), estas constelaciones de significado redefinen el campo de lo político. De hecho, las luchas de resistencia contra el acaparamiento de los bienes comunes no son simplemente luchas por recursos, son luchas por sentido: el sentido de la relación entre seres humanos y naturaleza, de la participación democrática y la autonomía, del bienestar y la dignidad.

A través de una revisión necesariamente parcial de datos etnográficos, con el fin de contribuir desde la antropología a la teorización de los comunes, en los apartados sucesivos busco esbozar la articulación entre los siguientes elementos: las nociones de propiedad, valor y bienestar que orientan la definición y la gestión de los bienes comunes a nivel comunitario; la construcción simultánea de un sentido de comunidad para y desde la protección y la gestión colectiva de tales bienes; los significados alternativos de soberanía que se construyen en el ejercicio de la gestión y la defensa de los bienes comunes. El papel de la comida en ontologías no-dualistas es el hilo conductor para abordar la relación entre soberanía alimentaria, soberanía territorial y derecho a los bienes comunes. 


\section{Las relaciones alimentarias en la constitución de lo distintamente humano: cuerpo, sociedad, conocimiento y territorio}

Además de su función obvia de asegurar la subsistencia, los alimentos son un medio complejo por el cual se crean, reproducen y transforman identidades, relaciones socioambientales y fenómenos culturales. Sin embargo, la aceptabilidad cultural de los alimentos, que es un aspecto del contenido normativo del derecho a la alimentación ${ }^{6}$, no va mucho más allá del reconocimiento de la variabilidad cultural de los hábitos y preferencias alimentarias. En discusiones recientes se ha recalcado que la aceptabilidad cultural implica también tener en cuenta valores no basados en la nutrición que se asocian a los alimentos y que contribuyen a la imagen completa del individuo y de la comunidad. Estos valores definen "no solo la salud física, sino también los aspectos emocionales, mentales y espirituales de la salud, la curación y la prevención de la enfermedad” (Kuhnlein 2009, 3). Las dinámicas profundamente sociales de la producción, preparación, intercambio y consumo de alimento son además un medio para concebir y relacionarse con el mundo. Para muchos pueblos amerindios, estas dinámicas están vinculadas con el mantenimiento de la vida en general. Es más, cuerpo, sociedad, conocimiento y territorio emergen simultáneamente del trabajo productivo y de cuidado, por lo tanto no es pertinente verlos como ámbitos separados.

Al observar que es esencialmente mediante las relaciones alimentarias que se construyen identidades, lazos de parentesco y relaciones de reciprocidad y dependencia, estas han sido consideradas como operadores fundamentales de la sociabilidad amerindia (Fausto 2007; Gow 1991; McCallum 2001, entre otros). En su clásico estudio de la alimentación en los Andes ecuatorianos, Mary Weismantel (1998) afirma que:

Los cuerpos están hechos de comida, y especialmente de diferentes cereales y tubérculos, cada uno de los cuales tiene su propio efecto

El derecho a la alimentación fue reconocido en la Declaración Universal de los Derechos Humanos de 1948 (art. 25) y consagrado en el Pacto Internacional de Derechos Económicos, Sociales y Culturales (art. 11): "El derecho a la alimentación adecuada se ejerce cuando todo hombre, mujer o niño, ya sea solo o en común con otros, tiene acceso físico y económico, en todo momento, a una alimentación adecuada o a medios para obtenerla" (PIDESC 1966). El derecho a una alimentación adecuada implica también que la alimentación debe poder ser aceptada culturalmente (Comité de Derechos Económicos, Sociales y Culturales. Observación general n. ${ }^{\circ} 12$ sobre el derecho a una alimentación adecuada, párrafos 6 y 7, 1999). Para ampliar, véase el informe del relator especial sobre el derecho a la alimentación, Olivier de Schutter: http://www.srfood.org/es/derecho-a-la-alimentacion. 
característico sobre el cuerpo humano. El comer cereales, sembrados por la propia unidad doméstica en su propia tierra, cosechados y procesados mediante el trabajo de la familia, da como resultado un cuerpo y una persona que son afectados directamente por la calidad del trabajo y las habilidades invertidas en la parcela. Aquellos que comen juntos en la misma casa, comparten la misma carne en un sentido completamente literal: están hechos de la misma sustancia. (89-90)

Una perspectiva similar se encuentra en varias culturas indígenas amazónicas, según las cuales la humanidad —en el sentido de condición inherente al ser humano- no está dada, sino que es una construcción frágil, incesantemente propensa a la desintegración que debe ser mantenida a través de procesos intencionales y constantes de "fabricación” (Descola y Pálsson 1996; Fausto 2007; Rival 2005; Santos-Granero 2012; Viveiros de Castro 1987, 1998). Según Viveiros de Castro (1987):

[T]al fabricación es concebida [...] como un conjunto sistemático de intervenciones sobre las sustancias que comunican el cuerpo con el mundo: fluidos corporales, alimentos, eméticos, tabaco, aceites y tintes vegetales. Las mutaciones corporales así producidas son la causa y el instrumento de transformaciones en términos de identidad social. Esto significa que no es posible una distinción ontológica [...] entre procesos fisiológicos y procesos sociológicos, a nivel del individuo ${ }^{7}$. (31)

En otras palabras, el cuerpo no es un soporte inerte sobre el cual se deposita lo social, sino que la formación del cuerpo y de la identidad social a través de sustancias es una y la misma cosa. Para la Gente de Centro ${ }^{8}$, los alimentos producidos por medio de una intervención sobre las sustancias forman gradualmente cuerpos dotados de los hábitos y las cualidades morales necesarias para vivir en sociedad. Las dietas marcan las diferentes etapas de la vida, desde antes del nacimiento; varían de acuerdo con el género, la posición social, las tareas o el estado de salud de una persona, e influyen en la constitución moral y los procesos

7 Estas ideas sugieren una ontología a la que Viveiros de Castro (2004) llama multinaturalista con el fin de diferenciarla de las ontologías multiculturalistas modernas: "Mientras estas últimas están fundadas en la implicación mutua de la unidad de la naturaleza y la multiplicidad de las culturas -la primera garantizada por la universalidad objetiva del cuerpo y la sustancia, la última generada por la particularidad subjetiva de espíritu y el significado- la concepción amerindia presume una unidad espiritual y una diversidad corpórea" (466).

8 La expresión Gente de Centro se refiere a un conjunto de grupos étnicos lingüísticamente diversos pero relativamente uniformes en el ámbito cultural, que incluye a uitotos, muinanes, boras, mirañas andokes, nonuyas y ocainas. El territorio ancestral de estos grupos comprende el interfluvio Putumayo-Caquetá. Actualmente los miembros de estas etnias se encuentran en distintas regiones de la Amazonía colombiana y peruana, además de otras regiones de Colombia. 
cognitivos de quien consume los alimentos y de sus parientes. El cumplimiento cabal de las dietas es un requisito imprescindible en el aprendizaje de saberes culturales expertos y las dietas específicas marcan la realización de labores como sembrar una chagra, ir de cacería o sostener la formación del feto durante el embarazo. Los procesos de transformación y las cualidades de los alimentos — su sabor (dulce, salado, picante) y textura (fina, efervescente, gruesa)—, además de afectar los procesos cognitivos, sirven para hablar del conocimiento (Micarelli 2015b). Decir que el conocimiento tiene sabor, o que el conocimiento verdadero se reconoce por su sabor, es más que una metáfora. Conocimiento y alimento comparten una misma sustancia de origen divino que actúa física y espiritualmente, manifestándose en la vitalidad de las personas, del grupo social y de su entorno. Conocimiento y alimento, manifestaciones del trabajo humano e incorporados simultáneamente, forman un cuerpo distintamente humano: "ensamblaje de afectos y formas de ser que constituyen un habitus" (Viveiros de Castro 2004, 475).

Entre la Gente de Centro, la idea de que los alimentos son el medio para incorporar y dar cuerpo a la identidad aparece frecuentemente en las evaluaciones de los cambios alimentarios y en el apego ideal a la alimentación tradicional. Una mujer uitoto, por ejemplo, se quejaba de que los niños de su comunidad nunca iban a pensar como verdaderos indígenas porque su dieta había sustituido los alimentos basados en los tubérculos por arroz. Otra mujer sostenía que la razón por la cual había podido mantener su cultura a pesar de haber vivido muchos años entre los blancos era haber sido alimentada cuando niña por su abuela, de acuerdo con los conocimientos de su linaje; estos saberes vuelven a tomar cuerpo cuando ella cultiva y comparte los productos de su chagra en el lugar donde se ha reasentado. Como sugiere Sutton (2001), al evocar un mundo de lugares, relaciones familiares, asociaciones agrícolas y conocimientos culturales, el alimento se convierte en un punto de identificación para aliviar la experiencia del desarraigo y reconstituir un sentido de integridad (wholeness). Al mismo tiempo, preparar y ofrecer alimentos culturalmente adecuados demuestra el conocimiento y el poder de una mujer y se vuelve el medio por el cual ella reafirma su posición social.

La relación de interdependencia entre las mujeres y sus cultivos se expresa a menudo a través del idioma del parentesco. Prender fogatas en la chagra para calentar los cultivos con el vapor de la olla — así como se calienta al niño en el vientre- hace que los cultivos "reconozcan a su mamá". Comer puede ser también un acto de aprecio hacia el alimento, que agrada a la cosa consumida y que, por ende, le otorga abundancia a quien la consumió, como sugiere la historia wari’ del espíritu del maíz recopilada por Conklin (1995): 
Hace mucho tiempo, un hombre caminaba a su chagra llevando una cesta de semillas de maíz para sembrar. Un grano de maíz cayó al suelo en el camino. El hombre no lo vio y siguió. La semilla de maíz comenzó a llorar como un niño. Otro hombre se acercó y lo encontró llorando en el suelo. Lo recogió y se lo comió. De este modo lo salvó, demostrando que sentía simpatía por él. El hombre que se comió la semilla sembró su chagra y dio grandes cantidades de maíz. El hombre que había dejado la semilla en el suelo plantó su campo, pero nada creció. (85)

Lo que llamamos naturaleza, para muchos pueblos amerindios es habitado por múltiples seres sobrenaturales con distintos dominios y potencias. Asociadas usualmente con lugares delimitados del territorio, como rocas, salados o manantiales, estas entidades son consideradas los “dueños” de los recursos (no objetos, sino sujetos) y se encargan de regular su aprovechamiento. Entre los nahuas de Pahuatlán (Puebla, México), Itekontlakuali, el “dueño de la comida”, beneficia a los humanos con una abundante cosecha siempre y cuando haya algo a cambio; si no se cumple con la vuelta, sobreviene el daño que se manifiesta como carencia o enfermedad (Acosta 2014, 225). En otras palabras, hay una relación clara entre el mantenimiento de este estatuto de intercambio y la salud. Para los embera de la vertiente pacífica colombiana, son los jaibaná, médicos y especialistas rituales, quienes se encargan de invocar a los dueños de los recursos con el fin de propiciar abundancia o ahuyentar una especie (Pardo 1987). Entre los miraña de la Amazonía colombiana la comida es controlada por los "dueños del mundo”, y su acceso es regulado por restricciones y obligaciones que deben ser tenidas en cuenta antes y después de la preparación y consumo de alimentos (Tapia 2009). Los sabedores deben negociar con estos dueños para obtener beneficios y, al mismo tiempo, asegurar la inmunidad frente a las enfermedades que pueden causar los recursos del monte. Para los uitoto, los principios que regulan la siembra de una chagra incluyen conocer el calendario ecológico y epidemiológico, elegir física y espiritualmente el terreno, hacer un convenio espiritual y mantener el diálogo con la naturaleza, controlar el trabajo por medio de las dietas, compartir los productos sin egoísmo para que "no le entre el mal espíritu”, tener disponibilidad de alimentos para la minga ${ }^{9} \mathrm{y}$ convidar con tiempo a los que van a trabajar (Acosta et al. 2011, 78). El cumplimiento de estas reglas implica la corresponsabilidad de las familias indígenas frente a sus espacios cultivados y tiene como fin asegurar no solo abundancia en la cosecha, sino también la buena salud del individuo, la sociedad y la naturaleza. Así que el saber culinario y el 
conocimiento inherente al manejo del mundo convergen y se ensamblan. Los rituales de baile - cuyo propósito primario es "curar el mundo"-son una de las formas con las que se ratifica el convenio con la naturaleza y se paga de vuelta a los dueños.

Desde una concepción del mundo que establece el estatuto de dependencia mutua entre humanos y no humanos, su reproducción y permanencia es posible solo gracias a la constante relación entre ambos (Acosta 2014) ${ }^{10}$. En una perspectiva compartida por muchas sociedades amazónicas (Griffiths 2002; Micarelli 2015a; Overing y Passes 2000), el elemento indispensable de esta relación es el trabajo. En las concepciones indígenas el trabajo no se refiere exclusivamente a las prácticas materiales de producción, sino que es el medio por el cual se forman de manera gradual hábitos y disposiciones sociales y se mantienen buenas relaciones con los otros, el entorno y los seres espirituales en la vida diaria. El trabajo se refiere al mantenimiento y la reproducción de las condiciones necesarias para la vida humana y del cosmos. Por medio del trabajo se conserva en constante circulación la energía vital — que es percibida como finita, escasa y distribuida desigualmente (Santos-Granero 2009)—, revelando una concepción del mundo en donde la acumulación es incompatible con la abundancia y esencialmente dañina (Micarelli 2015a). El trabajo para la Gente de Centro "se muestra en abundancia”: su demostración definitiva son los rituales de baile en los cuales grandes cantidades de comida son redistribuidas entre los convidados. A su vez, la capacidad para trabajar muestra de manera tangible el conocimiento de una persona, conocimiento validado por su contribución hacia el bienestar generalizado, que se convierte en una fuente de autoridad. Así que no solo el alimento, sino también el trabajo invertido en él confieren identidad individual y colectiva. Estas perspectivas revelan cómo la producción, preparación, intercambio y consumo de alimentos no son meros actos sociales, sino actos que establecen a la

10 Si bien se ha puesto mucho énfasis en la reciprocidad como modo predominante de compensación, esta no necesariamente es la única forma de interacción que se establece entre los humanos y los no-humanos. La evidencia etnográfica de las sociedades amerindias sugiere otros esquemas de interacción, que incluyen la rapacidad y la protección o cuidado (Descola y Pálsson 1996). La rapacidad se hace necesaria porque los dueños de los recursos son a menudo mezquinos y no quieren soltar sus bienes. En una concepción del mundo gobernada por lo que Santos-Granero (2009) llama la economía política de la vida, la rapacidad no tiene como fin la acumulación, sino alimentar los circuitos de reproducción de la vida. Por otro lado, el cuidado no necesariamente es simétrico o recíproco y está estrechamente relacionado con el control y la dependencia. Inspirados por el trabajo de Strathern $(1988,2005)$, algunos amazonistas ven en el cuidado el medio por el cual las personas hacen otras personas (Brightman, Fausto y Grotti 2016, 19). Estos diferentes modos de interacción -reciprocidad, rapacidad y cuidado- comparten la idea de que un mundo de igualdad es estéril y que la vida solo puede prosperar gracias al "deseo riesgoso por la diferencia" (Brightman, Fausto y Grotti 2016, 19; Overing 1981). 
sociedad y que definen la cualidad de las relaciones con el entorno. Su función es alimentar un entramado de relaciones de las cuales dependen la vida y la salud de todos; como declara el anciano uitoto cuya cita encabeza este texto, "ien el comer está la vida!”. Estas ideas se oponen al universalismo del concepto de seguridad alimentaria, cuya definición comúnmente aceptada es el estado en el cual "todas las personas tienen acceso físico, social y económico permanente a alimentos seguros, nutritivos y en cantidad suficiente para satisfacer sus requerimientos nutricionales y preferencias alimentarias, y así poder llevar una vida activa y saludable" (FAO 1996). Este concepto abstrae la alimentación de toda una serie de relaciones sociales y ambientales y ve el comer como acto individual, de un yo autónomo, desvinculado de los demás y del entorno. Además, el papel de la comida como eje portante de las ontologías indígenas amplifica el concepto de aceptabilidad cultural que informa el derecho a la alimentación. No es casual que las organizaciones indígenas de la Amazonía estén movilizando el concepto de soberanía alimentaria para cuestionar los programas asistencialistas de nutrición implementados desde el Estado y afianzar su autonomía ${ }^{11}$. Como lo recalca la presidente del Foro Permanente para los Asuntos Indígenas de la ONU, "[e]l derecho de los pueblos indígenas a la alimentación es inseparable de sus derechos a la tierra, el territorio, los recursos, la cultura y la autodeterminación” (Cunningham 2013, x).

\section{Territorio y soberanía}

En la cosmovisión indígena el territorio se compone de lo naturaly lo sobrenatural, representa el universo entero, lo que es UNO mismo, lo que está encima del suelo, sobre el suelo y debajo de él [...]

Más que el espacio físico, es donde se consigue el sustento para sobrevivir y donde se reproducen todas las relaciones de vida, existencia, crecimiento y realización espiritual. Indígena muinane

El paradigma de la soberanía alimentaria ha tenido que lidiar desde sus comienzos con la herencia moderna occidental del concepto de soberanía, originariamente la autoridad que emana de un soberano para ejercer el poder sobre sus súbditos. En las constituciones modernas, soberanía abarca tanto la autoridad 
del Estado-nación —-soberanía nacional- como el derecho de la ciudadanía a participar en el ejercicio del poder político — soberanía popular-, y se refiere al poder político supremo que corresponde a un Estado independiente en un ámbito territorial dado. El diálogo entre distintas culturas rurales, en una coyuntura marcada por las dinámicas de la economía global y el consecuente debilitamiento de la autoridad del Estado sobre el territorio, ha aportado significados más complejos al concepto de soberanía. En contra de la naturalización del territorio como equivalente al territorio nacional o estatal, las aproximaciones a la reforma rural bajo un enfoque territorial ${ }^{12}$, por ejemplo, reconocen que dentro y -a menudo-a través de las fronteras de los Estados-nacionales coexisten diferentes territorialidades, y por consiguiente que el ejercicio del poder político sobre el territorio no puede ser derecho exclusivo del Estado-nación. Además, el territorio se entiende no simplemente como un espacio geográfico delimitado, sino como una construcción sociohistórica que incorpora los saberes, las cosmovisiones y las distintas formas de gestión colectiva de los recursos desarrollados a lo largo del tiempo por las comunidades que lo habitan (Martínez y Rosset, en prensa).

Estas propuestas logran alterar el significado hegemónico del concepto de soberanía, pero solo parcialmente, ya que su modelo sigue basándose en el supuesto de una noción de autoridad informada por el marco político y legal que emana del Estado. Como señala el intelectual mohawk Taiaiake Alfred (2006), la descolonización real del concepto de soberanía requiere desconectar sus raíces occidentales y cuestionar los supuestos de una idea del poder modelada en términos del Estado. Su análisis del concepto de soberanía indígena brinda elementos valiosos en el intento de repensar la soberanía alimentaria y la soberanía en general. Para esto hay que comenzar por una consideración de las concepciones indígenas del orden natural y del territorio, que según Alfred son los referentes básicos para pensar el poder, la propiedad y la justicia en una perspectiva indígena. La perspectiva a la que se refiere Alfred aparece, con obvias variaciones, en numerosas culturas indígenas del continente (Surrallés y García 2005). Su elemento distintivo ha sido caracterizado por una vertiente teórica latinoamericana (De la Cadena 2010; Escobar 2010) como ontología relacional, por la cual el ser existe solo gracias a las relaciones que lo atraviesan y lo conectan con el mundo, superando de esta manera los dualismos humanidad/naturaleza y sujeto/objeto que caracterizan a la modernidad occidental. Las ontologías relacionales amerindias introducen una noción de territorio que excede en muchos sentidos las

\footnotetext{
12 Este tema, que ha adquirido particular relevancia en los acuerdos de paz firmados en Colombia en el 2016, ha comenzado a ser discutido desde hace dos décadas aproximadamente en Latinoamérica (véase Sepúlveda et al. 2003).
} 
definiciones occidentales dominantes de este concepto. Mientras que en estas últimas el territorio implica un área delimitada o una porción de espacio geográfico derivada de la pertenencia a un sujeto que ejerce en modo exclusivo su soberanía, en las cosmovisiones amerindias el territorio es un tejido de relaciones sociales en que el ser humano no es sino un actor entre muchos otros. Este tejido abarca diferentes dominios, tanto materiales como inmateriales, y estos dominios están sujetos a diferentes jurisdicciones. De aquí que su mantenimiento requiera la realización permanente de acuerdos entre todos sus constituyentes, humanos y no humanos. Más allá de una multidimensionalidad espacial, el territorio es también el locus de distintas temporalidades. Estas dimensiones son permeables; entre los pueblos andinos están atravesadas por el aliento vital, la sustancia del alma (samai en kechwa, samaña en aymara) que circula entre todos los seres vivos, la naturaleza y los sitios sagrados, conectando la realidad visible e invisible, el tiempo de los antepasados con el tiempo actual y el futuro posible. La relación con el territorio se configura en los términos del parentesco, como sustancia compartida (Uzendoski 2006).

El concepto de territorialización desarrollado por las ciencias sociales y humanas ha permitido revelar la complejidad de los procesos en que la presencia humana informa de manera reconocible un ámbito espacial, volviéndolo territorio. A través de este concepto se muestra cómo las prácticas humanas llevan a cargar de valores cada componente y el conjunto de un espacio geográfico, así que más que simples referentes espaciales, los elementos del territorio llegan a ser instrumentos para evocar y transmitir el conocimiento, la memoria y los valores (afectivos, económicos, jurídicos, ideológicos, políticos y religiosos) de quienes lo habitan. Sin embargo, en una ontología no dualista el territorio es mucho más que esto. Para los tikuna la tierra es el cuerpo de Ngutapa, el padre creador de los humanos ${ }^{13}$. En los mitos uitoto de Moniya Amena, la unión entre un dios y una mujer da origen al árbol de la abundancia del cual surgen simultáneamente la comida y el territorio. En otro mito muinane, el Creador es al principio solo un pensamiento, que adquiere cuerpo junto con la tierra a través de la palabra, florece y da frutos (Micarelli 2015b). Esta sustancia cosmogónica impregna la tierra y todo lo que vive en ella, manifestándose con particular fuerza en los sitios sagrados. Para muchos pueblos amazónicos la ley y el conocimiento emanan también de un origen divino y ambos son percibidos como inmutables; sin embargo, es responsabilidad de los humanos mantener el orden primordial en equilibrio, tarea que se realiza en la vida diaria de manera permanente. Las 
siguientes palabras de dos pensadores indígenas expresan la idea del territorio como tejido urdido por el conocimiento, reflejo tangible del actuar responsable de los humanos, y sugieren una noción de soberanía como cuidado o protección, en lugar de autoridad o control:

El pensamiento de los sabedores corre por el territorio y lo organiza, pensamiento que lo cobija todo y cuida el territorio. (Anciano uitoto, diario de campo, 1999)

Manejar el mundo es hacer que la vida sea posible, tanto la vida humana, como la vida de la naturaleza... Las personas dependemos de la selva. La selva depende de los humanos. Todo es relacionado: la tierra, la arena, la misma piedra. El conocimiento está allí. (Maximiliano García Makuna, en Rico y Decaillet 2010)

El concepto occidental de soberanía como derecho exclusivo sobre una porción del espacio geográfico que deriva de la pertenencia a un sujeto no podría estar más alejado del concepto indígena. El problema aquí es de traducción, no de ausencia. Los análisis antropológicos sobre la noción de propiedad en las sociedades indígenas han demostrado que su diferencia con las nociones occidentales radica en una particular construcción de la distinción entre sujeto y objeto y en las diferentes formas en que se establecen relaciones entre cosas y personas (Brightman, Fausto y Grotti 2016). Para las poblaciones indígenas de la Amazonía, por ejemplo, la apropiación del espacio no resulta en propiedad absoluta: "la propiedad para los indígenas tiene manifestaciones intangibles y espirituales y, aunque digna de protección, es inalienable y no puede pertenecer a ningún ser humano” (Posey y Dutfield 1996, 3).

[E]n lugar de tener los humanos meros derechos preferenciales sobre extensiones de tierra entendida como un objeto natural, la propiedad prolifera y enlaza lugares a través de relaciones entre personas no-humanas, con las cuales los seres humanos deben interactuar de diversas maneras, incluyendo la caza, la agricultura y el chamanismo [...]

Los derechos sobre la tierra también incluyen derechos territoriales, que son traducciones de las esferas de influencia de particulares unidades sociopolíticas. En términos indígenas, estos son articulados en términos de propiedad, pero la propiedad toma la forma de una red de relaciones: "Toda categoría de espacio... tiene la forma de una comunidad de seres relacionados juntos como posesores y poseídos” (Gow 1991, 80). (Brightman, Fausto y Grotti 2016, 22-23)

Estas perspectivas invitan a repensar la noción de soberanía más allá del concepto clásico del Estado-nación, en términos de las relaciones de "intra-dependencia” (De la Cadena 2015) que unen comunidades y territorios, relación que 
conlleva responsabilidad y de la que depende la pervivencia de ambos. He aquí que la ocupación del territorio no es necesariamente equivalente a propiedad o tenencia; más bien, son las personas quienes pertenecen a la tierra. Perspectivas similares son reivindicadas por los pueblos nativos norteamericanos. Corntassel (2012) refiere que en cherokee una relación sostenible puede expresarse a través de la palabra digadatsele'i, "pertenecemos el uno al otro" (96). Pertenecer el uno al otro significa ser responsable el uno del otro y del mundo natural. Su argumento para descolonizar el concepto de soberanía se sostiene precisamente en la idea de que mientras los derechos derivan de foros centrados en el Estado, las naciones indígenas actúan sobre sus responsabilidades inherentes y perdurables con el mundo natural, responsabilidades que se originan de sus relaciones con la tierra y que han existido mucho antes del desarrollo del sistema estatal (Corntassel 2012, 92). El investigador mohawk Taiaiake Alfred (2006) desarrolla estos conceptos de la siguiente manera:

Las filosofías indígenas se basan en la convicción de que la relación de los humanos con la tierra es principalmente una de mancomunidad. La tierra fue creada por un poder fuera de los seres humanos, y una relación justa con ese poder debe respetar el hecho de que los seres humanos no tomaron parte en hacer la tierra, por lo tanto, no tienen derecho a disponer de ella como mejor les parezca. La tierra es creada por otro orden de poder, por lo tanto la posesión por los seres humanos es antinatural e injusta. El principio de mancomunidad, que refleja una conexión espiritual con la tierra establecida por el Creador, da a los seres humanos responsabilidades especiales dentro de las áreas que ocupan, vinculándolos de una manera natural y sagrada a sus territorios [...]

Mientras que la relación humanos-tierra es estructurada por fuerzas mayores en la naturaleza fuera de la prerrogativa humana para el cambio, la relación humanos-instituciones implica una responsabilidad activa para los seres humanos de usar sus propios poderes de creación para lograr el equilibrio y la armonía. Las estructuras de gobierno y las instituciones sociales están diseñadas para empoderar a los individuos y reforzar la tradición para mantener el equilibrio encontrado en la naturaleza.

La soberanía, entonces, es una creación social. No es un fenómeno objetivo o natural, sino el resultado de elecciones hechas por hombres y mujeres, indicativo de una mentalidad ubicada en un orden social y político, en lugar de una fuerza natural creadora de dicho orden. La reificación de la soberanía en la política de hoy es el resultado del triunfo de un conjunto particular de ideas sobre otros — no más natural para el mundo que cualquier otro objeto creado por el hombre-. (470-471)

Las nociones indígenas de soberanía permean su percepción de la soberanía alimentaria. El Grupo de Trabajo sobre Soberanía Alimentaria de los Pueblos 
Indígenas de Canadá ${ }^{14}$, por ejemplo, afirma que la soberanía alimentaria depende de la interacción de una multitud de comunidades naturales que funcionan en conjunto. El alimento es un don sagrado y en este respecto el derecho a la alimentación no puede ser restringido por leyes, políticas e instituciones coloniales. La soberanía alimentaria se logra fundamentalmente cultivando la responsabilidad de nutrir relaciones saludables e interdependientes con la tierra, las plantas y los animales. Estas ideas contrastan drásticamente con la preocupante situación de desnutrición y malnutrición de los pueblos indígenas a escala global (Inter-Agency Support Group on Indigenous People's Issues 2014), resultado, entre otros, de la pérdida de tierra y territorio, la degradación ambiental, el deterioro de los sistemas productivos y las economías tradicionales, el conflicto armado, el desplazamiento y, ante todo, la expansión del sistema económico y alimentario colonial y neoliberal. Tal vez por estas mismas razones, y por configurar un sentido intrínseco de la identidad y la dignidad, el ideal de la soberanía alimentaria adquiere una fuerza significativa y provee el marco para negociar reformas políticas y afirmar el derecho a la autodeterminación.

\section{Conclusiones}

¿De qué maneras las perspectivas indígenas aquí presentadas pueden contribuir a las luchas de los movimientos sociales para alcanzar la soberanía alimentaria? Para comenzar, como señala Marisol de la Cadena (2010, 2015), la noción de representación política difiere radicalmente si el marco ontológico de referencia es un mundo ordenado por prácticas biopolíticas que conciben la vida humana discontinua de aquello que esas mismas prácticas definen como naturaleza, o un mundo en el que seres humanos y otros-que-humanos están inherentemente conectados en relaciones de cuidado mutuo. En una ontología relacional, las entidades emergen de la relación: "son intrarrelacionadas en vez que interrelacionadas” (De la Cadena 2015, 32). En kechwa esta práctica de intrarrelación, que no es altruismo sino una obligación, se llama uyway: cuidar, nutrir, criar (De la Cadena 2015, 103). Gestada en una ontología relacional, la práctica política que proponen los movimientos indígenas es "una práctica política diferente, plural no porque esté marcada por género, raza, etnicidad o sexo (como sostiene el

14 Working Group on Indigenous Food Sovereignty (WGIFS): https://www.indigenousfoodsystems. org/about. 
multiculturalismo), sino porque conjura a los no humanos como actores en la arena política” (De la Cadena 2010, 334).

La jurista Maria Rosaria Marella (2016) nos ofrece un ejemplo de cómo una ontología relacional puede ser movilizada estratégicamente para la protección de los bienes comunes y de cuáles son sus implicaciones para transformar el marco legal:

En el marco epistemológico de la dicotomía sujeto/objeto los bienes comunes son por lo general localizados dentro de este último extremo como objetos. Una solución alternativa para los bienes comunes puede ser convertir "el objeto" en una persona jurídica, es decir, en "sujeto legal”. Esta es la situación jurídica que ha sido reconocida recientemente a un río, el río Whanganui en Nueva Zelanda, en virtud de un acuerdo entre el Gobierno nacional y la población maorí. Sobre la base de una tradición indígena que identifica el río con la misma población, el Whanganui iwi (I am the river and the river is me), el acuerdo reconoce el río como Te Awa Tupua, una entidad jurídica autónoma. En términos legales, las responsabilidades de gestión se basan en los poderes de los representantes legales del río. (S. p.)

Un enfoque no naturalista para calificar los comunes permite conceptualizarlos no como bienes, sino como un conjunto de sistemas sociales (De Angelis 2017). Además, al romper el dualismo humanidad/naturaleza es posible entender la reproducción de sistemas sociales como parte de la reproducción de ecosistemas, y viceversa. Emergiendo de un proceso de construcción simultánea, sistemas sociales y ecosistemas son por lo tanto inseparables; esta unión establece un nuevo sujeto de derecho, colectivo y socionatural.

Además de una conceptualización no naturalista, hay un elemento adicional que puede orientar una lectura renovada de los comunes: la dignidad. En el momento de la firma del acuerdo, los representantes maoríes expresaron: "por fin mantenemos nuestra dignidad”. Apelar a la dignidad, en el sentido del valor inherente al ser humano, permea las luchas por la soberanía. Esto nos invita a preguntar cuál es la concepción de humanidad que subyace a esta y qué consideramos que es distintivamente humano. Estas preguntas deben informar nuestro pensamiento sobre la ley.

Frente a la multiplicación de formas de despojo y desterritorialización, la defensa de los comunes implica la reivindicación de nociones y prácticas distintas de la soberanía, con las cuales los movimientos indígenas y campesinos desafían la agresión sobre formas de vida locales, afirman derechos colectivos e imaginan alternativas políticas y económicas sostenibles. Estas luchas defienden también una noción distinta de bienestar, expresada a menudo en América 
Latina como "buen vivir". La defensa de los comunes como componente imprescindible de la soberanía alimentaria insiste en el papel de las relaciones alimentarias en la reproducción del tejido socionatural material y simbólico del cual depende la pervivencia de los humanos y de su entorno al mismo tiempo. El cuidado de estas relaciones es lo que hace posible la reproducción de la vida y la identidad, fundando la soberanía alimentaria y la soberanía en general en un ejercicio de responsabilidad.

Si, como ha recalcado el relator especial para el derecho a la alimentación de las Naciones Unidas, Olivier de Schutter (2014), la plena realización del derecho a la alimentación depende de la soberanía alimentaria, la soberanía alimentaria depende de los comunes: agua, semillas, paisajes biodiversos y conocimientos agredidos por la privatización neoliberal. La realización del derecho a la alimentación implica proteger y afianzar las relaciones de mancomunidad entre comunidades y territorios que garantizan la sostenibilidad y de las que emanan mosaicos de soberanía. A través de estas relaciones, el sentido de lo que nos hace distintamente humanos se multiplica, y es con esta pluralidad que toda noción de bienestar, salud, dignidad y derechos tiene que dialogar.

\section{Referencias}

Acosta, Luis Eduardo, Mónica Natalia Pérez Rúa, Luis Arcángel Juragaro, Honorio Nonokudo Faribiaño, Gentil Sánchez, Ángel María Zafiama Piñero, Juan Bosco Tejada Martínez, Osias Levi Cobete, Martin Efaiteke, Jeremias Farekade, Henry Giagrekudo, Simón Neikase. 2011. La chagra en La Chorrera. Los retos de las nuevas generaciones para las prácticas culturales y los saberes tradicionales asociados a la biodiversidad. Leticia: Sinchi; AZICATCH.

Acosta Márquez, Eliana. 2014. "Cuando el maíz es Itekontlakuali: el 'dueño de la comida’. Un acercamiento a la economía ritual de los nahuas de Pahuatlán, Puebla”. Cuicuilco 60: 223-238.

Alfred, Taiaiake. 2006. “Sovereignty”. En Sovereignty Matters: Locations of Contestation and Possibility in Indigenous Struggles for Self-Determination, editado por Joanne Barker, 35-50. Lincoln: University of Nebraska Press.

Appadurai, Arjun. 1986. The Social Life of Things. Commodities in Cultural Perspective. Cambridge: Cambridge University Press.

Barth, Fredrik. 1987. Cosmologies in the Making: A Generative Approach to Cultural Variation in Inner New Guinea. Nueva York: Cambridge University Press. 
Brightman, Mark, Carlos Fausto y Vanessa Grotti. 2016. "Altering Ownership in Amazonia”. En Ownership and Nurture: Studies in Native Amazonia Property Relations, editado por Mark Brightman, Carlos Fausto y Vanessa Grotti, 1-35. Nueva York; Oxford: Berghahn.

Conklin, Beth. 1995. “Thus Are Our Bodies, Thus Was Our Custom': Mortuary Cannibalism in an Amazonian Society”. American Ethnologist 22 (1): 75-101.

Corntassel, Jeff. 2012. "Re-envisioning Resurgence: Indigenous Pathways to Decolonization and Sustainable Self-Determination”. Decolonization: Indigeneity, Education \& Society 1 (1): 86-101.

Cunningham, Myrna. 2013. Prefacio a Indigenous People Food Systems and Well Being. Interventions and Policies for Healthy Communities, editado por Harriet Kuhnlein, Bill Erasmus, Dina Spigelski y Bárbara Burlingame, IX-X. Roma: FAO; CINE.

De Angelis, Massimo. 2010. "On the Commons: A Public Interview with Massimo de Angelis and Stavros Stavrides”. E-Flux Journal 17. http://www.e-flux.com/journal/17/67351/on-thecommons-a-public-interview-with-massimo-de-angelis-and-stavros-stavrides/.

-. 2017. Omnia Sunt Communia. On the Commons and the Transformation to Postcapitalism. Londres: Zed Books.

De la Cadena, Marisol. 2010. "Indigenous Cosmopolitics in the Andes: Conceptual Reflections beyond 'Politics'”. Cultural Anthropology 25 (2): 334-370.

-. 2015. Earth Beings: Ecologies of Practice across Andean Worlds. Durham, NC: Duke University Press.

De Schutter, Olivier. 2014. "The Transformative Potential of the Right to Food. Final Report Drawing Conclusions from his Mandate”. http://www.srfood.org/images/stories/pdf/officialreports/20140310_finalreport_es.pdf.

Descola, Philippe y Gísli Pálsson, eds. 1996. Nature and Society: Anthropological Perspectives. Londres; Nueva York: Routledge.

Escobar, Arturo. 2010. “Latin America at a Crossroads”. Cultural Studies 24 (1): 1-65.

FAO. 1996. Cumbre Mundial sobre la Alimentación. Consultado el 11 de octubre del 2017. http://www.fao.org/docrep/014/al936s/al936s00.pdf.

Fausto, Carlos. 2007. "Feasting on People: Eating Animals and Humans in Amazonia”. Current Anthropology 48 (4): 497-530.

Gow, Peter. 1991. Of Mixed Blood: Kinship and History in Peruvian Amazonia. Oxford: Clarendon Press.

Graeber, David. 2001. Toward an Anthropological Theory of Value. The False Coin of Our Own Dreams. Nueva York: Palgrave.

Griffiths, Thomas. 2002. "Finding One’s Body: Relationships between Cosmology and Work in North-West Amazonia”. En Beyond the Visible and the Material. The Amerindianization of Society in the Work of Peter Rivière, editado por Laura Rival y Neil L. Whitehead, 247-262. Oxford: Oxford University Press.

Gudynas, Eduardo. 2010. "La senda biocéntrica: valores intrínsecos, derechos de la naturaleza y justicia ecológica”. Tabula Rasa 13: 45-71. 
Inter-Agency Support Group on Indigenous People's Issues. 2014, "The Health of Indigenous People”. Documento temático para la preparación de 2014 World Conference on Indigenous Peoples. http://www.un.org/en/ga/president/68/pdf/wcip/IASG\%20Thematic\%20 Paper\%20-\%20Health\%20-\%20rev1.pdf.

Kuhnlein, Harriet V. 2009. “Why Are Indigenous Peoples’ Food Systems Important and Why do They Need Documentation?”. En Indigenous Peoples’ Food Systems: The Many Dimensions of Culture, Diversity and Environment for Nutrition and Health, editado por Harriet V. Kuhnlein, Bill Erasmus y Dina Spigelski, 1-7. Roma; Quebec: Food and Agriculture Organization of the United Nations; Centre for Indigenous Peoples' Nutrition and Environment.

LVC. 1996. "The Right to Produce and Access to Land”. Roma, World Food Summit, 11-17 de noviembre. www.acordinternational.org/silo/files/decfoodsov1996.pdf.

—. 2013. "El llamado de Yakarta”. VI Conferencia de la Vía Campesina. 9-13 de junio, Yakarta.

-. 2016. "There Can Be no Declaration on the Rights of Peasants without the Right to Land". www.eurovia.org/there-can-be-no-declaration-on-the-rights-of-peasants-without-theright-to-land/.

Marella, Maria Rosaria, ed. 2012. Oltre il pubblico e il privato. Per un diritto dei beni comuni. Verona: Ombre Corte.

-. 2016. "The Commons as a Legal Concept”. Law Critique 27 (3): 299-310.

Martínez, María Elena y Peter Rosset. (En prensa). "Diálogo de saberes en La Vía Campesina: soberanía alimentaria y agroecología”. En Soberanía alimentaria: prácticas y saberes locales para un movimiento global contrahegemónico, editado por Giovanna Micarelli y Luciana Jacob. Bogotá: Editorial Pontificia Universidad Javeriana.

Martínez-Alier, Joan. 2002. The Environmentalism of the Poor: A Study of Ecological Conflicts and Valuation. Cheltenham: Edward Elgar.

Martínez-Alier, Joan, Giuseppe Munda y John O’Neill. 1998. “Weak Comparability of Values as a Foundation for Ecological Economics”. Ecological Economics 26: 277-286.

McCallum, Cecilia. 2001. Gender and Sociality in Amazonia: How Real People Are Made. Oxford: Berg.

Micarelli, Giovanna. 2015a. "Divine Banknote: The Translation of Project Money into Public Wealth”. En Images of Public Wealth: Property, Identity, and Well-being in Native Amazonia, editado por Fernando Santos-Granero, 161-187. Tucson: University of Arizona Press.

-. 2015b. "Finding the Taste of Knowledge: The Orphan in Indigenous Epistemologies". Tipití: Journal of the Society for the Anthropology of Lowland South America 13 (2): 74-90.

Nyéléni. 2007. Foro para la Soberanía Alimentaria. Sélingué, 23-27 de febrero, Mali. https:// nyeleni.org/DOWNLOADS/Nyelni_SP.pdf.

O’Connor, Martin y Clive Spash, eds. 1999. Valuation and the Environment: Theory, Methods and Practice. Cheltenham: Edward Elgar.

Ostrom, Elinor. 1990. Governing the Commons. The Evolution of Institutions for Collective Actions. Cambridge: Cambridge University Press. 
Overing, Joan. 1981. "Review Article: Amazonian Anthropology”. Journal of Latin American Studies 13 (1): 151-164.

Overing, Joan y Alan Passes, eds. 2000. The Anthropology of Love and Anger. The Aesthetics of Conviviality in Native Amazonia. Londres: Routledge.

Pardo, Mauricio. 1987. El convite de los espíritus. Quibdó: Ediciones Centro de Pastoral Indigenista.

Parry, Jonathan y Maurice Bloch, eds. 1989. Money and the Morality of the Exchange. Cambridge: Cambridge University Press.

Porto-Gonçalves, Carlos Walter. 2009. "De saberes y de territorios: diversidad y emancipación a partir de la experiencia latino-americana”. Revista de la Universidad Bolivariana 8 (22): $121-136$.

Posey, Darrell y Graham Dutfield. 1996. Beyond Intellectual Property: Toward Traditional Resource Right for Indigenous People and Local Communities. Ottawa: International Development Research Center.

Quilligan, James. 2012. "Why Distinguish Common Goods from Public Goods?”. En The Wealth of the Commons. A World beyond Market and State, editado por David Bollier y Silke Helfrich. Amherst: Levellers Press.

Ribeiro, Silvia. 2007. "En busca de la autonomía y la soberanía alimentaria”. Consultado el 17 de julio del 2017. https://nyeleni.org/spip.php?article297.

Rico, Diana y Richard Decaillet, dirs. 2010. Los conocimientos tradicionales de los chamanes jaguares de Yuruparí. Documental. https://www.youtube.com/watch?v=Gq4dg-KmSms.

Rival, Laura. 2005. “Introduction: What Constitutes a Human Body in Native Amazonia?”. Tipití: Journal of the Society for the Anthropology of Lowland South America 3 (2): 105-110.

Rodotà, Stefano. 2012. Il diritto ad avere diritti. Roma: Laterza.

Santos, Abel. 2013. "Percepción tikuna de Naane y Naüne: territorio y cuerpo". Tesis de Maestría en Estudios Amazónicos, Universidad Nacional de Colombia, sede Amazonía.

Santos-Granero, Fernando. 2009. Vital Enemies: Slavery, Predation, and the Amerindian Political Economy of Life. Austin: University of Texas Press.

-. 2012. "Beinghood and People-Making in Native Amazonia: A Constructional Approach with a Perspectival Coda”. HAU-Journal of Ethnographic Theory 2 (1): 181-211.

Sepúlveda, Sergio, Adrián Rodríguez, Rafael Echeverri y Melania Portilla. 2003. El enfoque territorial de desarrollo rural. San José: IICA.

Stark, David. 2011. The Sense of Dissonance: Accounts of Worth in Economic Life. Princeton: Princeton University Press.

Strathern, Marilyn. 1988. The Gender of the Gift: Problems with Women and Problems with Society in Melanesia. Berkeley: University of California Press.

-. 2005. Kinship, Law and the Unexpected: Relatives Are always a Surprise. Cambridge: Cambridge University Press. 
Surrallés, Alexandre y Pedro García Hierro, eds. 2005. The Land Within: Indigenous Territory and Perception of the Environment. Copenhagen: IWGIA.

Sutton, David. 2001. Remembrance of Repast. An Anthropology of Food and Memory. Nueva York: Berg Publishing.

Tapia, Carmensusana. 2009. “Améjimínaa majcho: 'La comida de nuestra gente’. Etnografía de la alimentación entre los miraña”. Culturales 5 (9): 39-72.

Uzendoski, Michael. 2006. "El regreso de Jumandy: historicidad, parentesco y lenguaje en Napo”. Iconos. Revista de Ciencias Sociales 26: 161-172.

Viveiros de Castro, Eduardo. 1987. “A fabricação do corpo na sociedade xinguana”. En Sociedades indígenas e indigenismo no Brasil, editado por João Pacheco de Oliveira Filho, 31-41. Río de Janeiro: Marco Zero; UFRJ.

-. 1998. "Cosmological Deixis and Amerindian Perspectivism". Journal of the Royal Anthropological Institute 4 (3): 469-488.

-. 2004. "Exchanging Perspectives. The Transformation of Objects into Subjects in Amerindian Ontologies”. Common Knowledge 10 (3): 463-484.

Weismantel, Mary. 1998. "Viñachina: hacer guaguas en Zumbagua, Ecuador”. En Gente de carne y hueso: las tramas del parentesco en los Andes, editado por Denise Y. Arnold, 83-96. La Paz: ILCA/Ciase. 